\title{
Nonlinear simulations of magnetic Taylor-Couette flow with current- free helical magnetic fields
}

\author{
J. Szklarski ${ }^{\star}$ and G. Rüdiger \\ Astrophysikalisches Institut Potsdam, An der Sternwarte 16, D-14482 Potsdam, Germany \\ Received July 2, 2006, accepted later \\ Published online more later
}

Key words methods: numerical - magnetic fields - magnetohydrodynamics (MHD)

The magnetorotational instability (MRI) in cylindrical Taylor-Couette flow with external helical magnetic field is simulated for infinite and finite aspect ratios. We solve the MHD equations in their small Prandtl number limit and confirm with timedependent nonlinear simulations that the additional toroidal component of the magnetic field reduces the critical Reynolds number from $O\left(10^{6}\right)$ (axial field only) to $O\left(10^{3}\right)$ for liquid metals with their small magnetic Prandtl number. Computing the saturated state we obtain velocity amplitudes which help designing proper experimental setups. Experiments with liquid gallium require axial field $\sim 50$ Gauss and axial current $\sim 4 \mathrm{kA}$ for the toroidal field. It is sufficient that the vertical velocity $u_{z}$ of the flow can be measured with a precision of $0.1 \mathrm{~mm} / \mathrm{s}$.

We also show that the endplates enclosing the cylinders do not destroy the traveling wave instability which can be observed as presented in earlier studies. For TC containers without and with endplates the angular momentum transport of the MRI instability is shown as to be outwards.

(c) 2006 WILEY-VCH Verlag GmbH \& Co. KGaA, Weinheim

\section{Introduction}

The magnetorotational instability (MRI) has been formulated long time ago (Velikhov 1959) but recently it become of particular interest for astrophysics due to recognition of its importance as the source of turbulence in accretion disks with Keplerian rotation (Balbus \& Hawley 1991). One of the most convenient models for MRI is magnetohydrodynamical cylindrical Taylor-Couette flow, i.e. motion of liquid metal between two concentric differentially rotating cylinders under imposed external magnetic field along the axis (Rüdiger \& Zhang 2001; Ji, Goodman \& Kageyama 2001; Willis \& Barenghi 2002). Under the influence of a purely axial field MRI operates for magnetic Reynolds number $\mathrm{Rm}=\Omega_{\text {in }} R_{\text {in }}\left(R_{\text {out }}-R_{\text {in }}\right) / \eta$ of order $O(10)$ and has never been observed in an experiment due to very small Prandtl number for laboratory liquids (see Table 1) which results in need of high critical Reynolds numbers of order $10^{6}$. Recently it has been shown by Hollerbach \& Rüdiger (2005) and Rüdiger et al. (2005) that a current-free external toroidal magnetic field in addition to the usual axial field can reduce the critical Reynolds number to $O\left(10^{3}\right)$ and therefore it makes it much easier to design a MRI experiment due to slower rotation.

The aim of the presented paper is to compute the amplitudes of velocities and the frequencies in a nonlinear saturated state under the influence of the endplates covering the cylinders.

The fluid confined between the cylinders is assumed to be incompressible and dissipative with the kinematic vis-

\footnotetext{
^ Corresponding author: jszklarski@aip.de
}

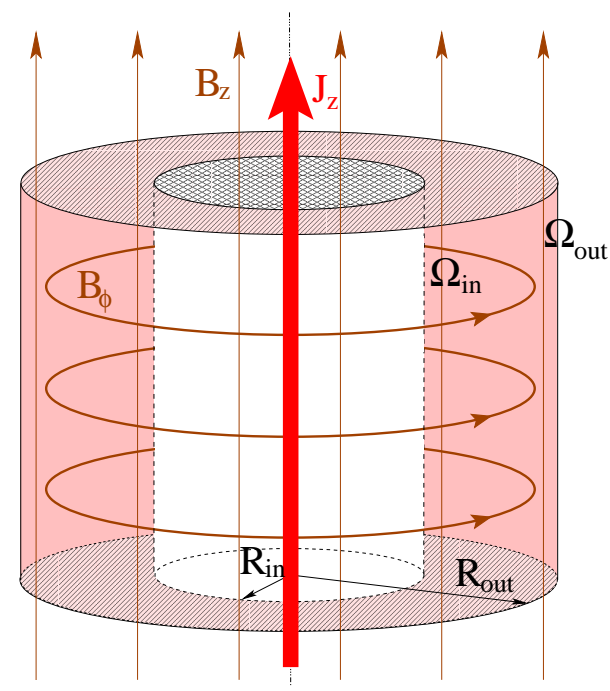

Fig. 1 The geometry of the problem - two concentric cylinders with radii $R_{\text {in }}=4 \mathrm{~cm}, R_{\text {out }}=8 \mathrm{~cm}$ rotating with $\Omega_{\text {in }}, \Omega_{\text {out }} . B_{z}$ and $B_{\phi}$ are the external magnetic fields. $B_{\phi}$ is due to an axial current inside the inner cylinder. In the PROMISE experiment cylinders have finite length of $H=40 \mathrm{~cm}(\Gamma=10)$ and are covered with endplates. The liquid is a mixture of gallium, indium and tin (Stefani et al. 2006).

cosity $\nu$ and the magnetic diffusivity $\eta$. Derived from the conservation of angular momentum the rotation law $\Omega(R)$ in the fluid is

$\Omega(R)=a+\frac{b}{R^{2}}$ 
Table 1 Physical properties of liquid metals which are suitable for the MRI experiment. A star denotes the PROMISE mixture.

\begin{tabular}{lcccc}
\hline & $\rho\left[\mathrm{g} / \mathrm{cm}^{3}\right]$ & $\nu\left[\mathrm{cm}^{2} / \mathrm{s}\right]$ & $\eta\left[\mathrm{cm}^{2} / \mathrm{s}\right]$ & $\mathrm{Pm}$ \\
\hline sodium & 0.9 & $7.1 \cdot 10^{-3}$ & $0.8 \cdot 10^{3}$ & $0.9 \cdot 10^{-5}$ \\
gallium & 6.0 & $3.2 \cdot 10^{-3}$ & $2.1 \cdot 10^{3}$ & $1.5 \cdot 10^{-6}$ \\
gallium $^{*}$ & 6.4 & $3.4 \cdot 10^{-3}$ & $2.4 \cdot 10^{3}$ & $1.4 \cdot 10^{-6}$ \\
\hline
\end{tabular}

with

$a=\frac{\hat{\mu}-\hat{\eta}^{2}}{1-\hat{\eta}^{2}} \Omega_{\mathrm{in}}, \quad b=\frac{1-\hat{\mu}}{1-\hat{\eta}^{2}} R_{\mathrm{in}}^{2} \Omega_{\mathrm{in}}$,

where $\hat{\mu}=\Omega_{\text {out }} / \Omega_{\text {in }}$. After the Rayleigh stability criterion, i.e. $\partial_{R}\left(R^{2} \Omega\right)^{2}>0$, the flow is hydrodynamically stable for $\hat{\mu}>\hat{\eta}^{2}$, i.e. $\hat{\mu}>0.25$ for our geometry. In order to isolate MRI we are mainly interested in hydrodynamically stable regimes so that $\hat{\mu}>\hat{\eta}^{2}$ should be fulfilled.

\section{Equations and numerical model}

The dimensionless incompressible MHD equations are

$\partial_{t} \boldsymbol{u}+(\boldsymbol{u} \cdot \nabla) \boldsymbol{u}=-\nabla p+\nabla^{2} \boldsymbol{u}+\frac{\mathrm{Ha}^{2}}{\mathrm{Pm}}(\operatorname{rot} \boldsymbol{B}) \times \boldsymbol{B}$,

$\partial_{t} \boldsymbol{B}=\frac{1}{\mathrm{Pm}} \nabla^{2} \boldsymbol{B}+\operatorname{rot}(\boldsymbol{u} \times \boldsymbol{B})$,

with $\operatorname{div} \boldsymbol{u}=\operatorname{div} \boldsymbol{B}=0$ and with the Hartmann number

$\mathrm{Ha}=B_{0} \sqrt{\frac{R_{\text {in }}\left(R_{\text {out }}-R_{\text {in }}\right)}{\mu_{0} \rho \nu \eta}}$.

The magnetic Prandtl number is $\operatorname{Pm}=\nu / \eta . D=R_{\text {out }}-$ $R_{\text {in }}$ is used as the unit of length, $\nu / D$ as the unit of velocity and $B_{0}$ as the unit of magnetic fields. The Reynolds number Re is defined as $\operatorname{Re}=\Omega_{\text {in }} R_{\text {in }} D / \nu$.

For laboratory liquids like gallium, the magnetic Prandtl number is very small (see Table 1 so the magnetic diffusion time is much shorter than other time scales and fluctuations $\boldsymbol{b}$ of the field $\boldsymbol{B}_{\mathbf{0}}+\boldsymbol{b}$ are also much smaller than the applied field $\boldsymbol{B}_{0}$. As a result the $\boldsymbol{b}$ adjusts instantaneously to the velocity $\boldsymbol{u}$ and the quasi-static approximation can be used (Roberts 1967; Zikanov \& Thess 1998; Youd \& Barenghi 2006).

With $\boldsymbol{B} \rightarrow \boldsymbol{B}_{\mathbf{0}}+\mathrm{Pm} \boldsymbol{b}$ and $\mathrm{Pm} \rightarrow 0$ one finds the equation system

$\partial_{t} \boldsymbol{u}+(\boldsymbol{u} \cdot \nabla) \boldsymbol{u}=-\nabla p+\nabla^{2} \boldsymbol{u}+\mathrm{Ha}^{2}(\nabla \times \boldsymbol{b}) \times \boldsymbol{B}_{\mathbf{0}}$,

$\nabla^{2} \boldsymbol{b}=-\operatorname{rot}\left(\boldsymbol{u} \times \boldsymbol{B}_{\mathbf{0}}\right)$

and $\operatorname{div} \boldsymbol{u}=\operatorname{div} \boldsymbol{b}=0$. The external field is

$\boldsymbol{B}_{\mathbf{0}}=\left(0, B_{\phi}, B_{0}\right)=B_{0}\left(0, \frac{\beta R_{\text {in }}}{R}, 1\right)$,

where the parameter $\beta$ denotes the ratio of toroidal field at the inner cylinder to the constant axial field $B_{0}$. Except along the axis the magnetic field is current-free.

We simulate the axisymmetric 2D flow in cylindrical coordinates $(R, \phi, z)$ with the numerical code of A. Youd (see Youd \& Barenghi 2006 for details). The code has been modified in order to handle periodic boundary conditions in a way suitable for our needs, the toroidal field was added and different boundary conditions on the endplates were applied.

Equations (5) are solved with a finite-difference method in the $R-z$ plane in a streamfunction-vorticity formulation, i.e.

$$
\begin{aligned}
\partial_{t} u_{\phi} & =\left(\nabla^{2}-R^{-2}\right) u_{\phi}+[\boldsymbol{u} \times(\operatorname{rot} \boldsymbol{u})]_{\phi}+\mathrm{Ha}^{2} \partial_{z} B_{\phi} \\
\partial_{t} \omega_{\phi} & =\left(\nabla^{2}-R^{-2}\right) \omega_{\phi}- \\
& {[\operatorname{rot}(\boldsymbol{\omega} \times \boldsymbol{u})]_{\phi}+\mathrm{Ha}^{2}\left[\operatorname{rot}\left(\boldsymbol{j} \times \boldsymbol{B}_{\mathbf{0}}\right)\right]_{\phi}, }
\end{aligned}
$$

with 'Poisson equations' for streamfunction, magnetic field and the current

$$
\begin{aligned}
& \left(-R^{-1}\left(\partial_{z z}+\partial_{r r}\right)+R^{-2} \partial_{r}\right) \psi=\omega_{\phi} \\
& \left(\nabla^{2} \boldsymbol{B}\right)_{\phi}=-\partial_{z}\left(u_{\phi}-u_{z} \beta B_{0} \frac{R_{\text {in }}}{R}\right)+\partial_{R} u_{R} \beta B_{0} \frac{R_{\text {in }}}{R} \\
& \left(\nabla^{2} \boldsymbol{j}\right)_{\phi}=-\partial_{z z}\left(u_{R}\right)+\partial_{R} \frac{\partial_{R}\left(R u_{R}\right)}{R},
\end{aligned}
$$

where the streamfunction $\psi$ is defined by $u_{R}=$ $-(1 / R) \partial_{z} \psi, u_{z}=(1 / R) \partial_{R} \psi$ and vorticity $\boldsymbol{\omega}=\operatorname{rot}(\boldsymbol{u})$.

No-slip boundary conditions for the velocity on the walls and the endplates are used, so that $u_{r}=0$ at $R=$ $R_{\text {in }}, R_{\text {out }}$ and $u_{z}=0$ at $z=0, H$. Therefore $\psi=0$ at $R=R_{\text {in }}, R_{\text {out }}$ and $z=0, H$. Using $(8)_{1}$ we obtain $\omega_{\phi}=$ $-(1 / R) \partial_{r r} \psi$ at $R=R_{\text {in }}, R_{\text {out }}$ and $\omega_{\phi}=-(1 / R) \partial_{z z} \psi$ at $z=0, H$. Boundary conditions for $u_{\phi}$ at the walls are determined by $\operatorname{Re}$ and $\hat{\mu}$ and at the endplates by their rotation properties - fixed or rotating as rigid body with $\Omega_{\text {out }}$ or $\Omega_{\text {in }}$.

The magnetic boundary conditions depend on the electrical properties of the walls. For perfectly conducting cylinders we assume infinite conductivity so that with $\boldsymbol{J}=$ $\sigma(\boldsymbol{E}+\boldsymbol{u} \times \boldsymbol{b})$ and $E_{\phi}=E_{z}=0$ for the walls we get $R^{-1} b_{\phi}+\partial_{R} b_{\phi}=0, \quad j_{\phi}=0 \quad$ at $\quad R=R_{\text {in }}, R_{\text {out }}$.

In the present paper only insulating endplates are considered and the BCs for magnetic field are obtained by applying the so called pseudo-vacuum approximation for which $b_{r}=$ $b_{\phi}=\partial_{z} b_{z}=0$. Then

$b_{\phi}=\partial_{z} j_{\phi}=0$

at $z=0, H$. We use grid resolution $N_{R} \times N_{z}=80 \times 800$ (and $40 \times 400$ for comparison, the difference in obtained values is always less than couple of percent). The time-step is constant with $\mathrm{d} t=10^{-5}$ or $\mathrm{d} t=10^{-4}$.

\section{Infinite container}

First we have performed simulations for periodic vertical boundary conditions in order to compare the critical Reynolds numbers with those presented in Rüdiger et al. (2005) as well as to compute for the first time amplitudes of velocities in the nonlinear saturated state. For infinite cylinders and $\hat{\mu}=0.27$ the flow is always hydrodynamically stable, with external axial magnetic field it looses the stability for large Reynolds number of order $O\left(10^{6}\right)$ but the 



Fig. 2 Snapshots of contour lines of the streamfunction: dashed lines represent counterclockwise rotation, solid lines clockwise rotation. $\mathrm{Ha}=9.5, \mathrm{Re}=900, \hat{\mu}=0.27, \beta=4$. The graph is not at scale.



Fig. 3 Frequency of the traveling wave as a function of rotation ratio $\hat{\mu}$ for infinite cylinders, $\mathrm{Ha}=9.5, \mathrm{Re}=$ $900, \beta=4$. Here and below the units are scaled according to physical properties of gallium.

additional toroidal current-free field can reduce this number to $O\left(10^{3}\right)-$ for $\beta=3,4$ the critical $\operatorname{Re}_{\text {crit }}$ is 1160 and 842 , resp.

In the simulations the length of the periodic cylinders was chosen to be three times the wavelength obtained with linear analysis. The agreement between the previous and presented results is rather good.

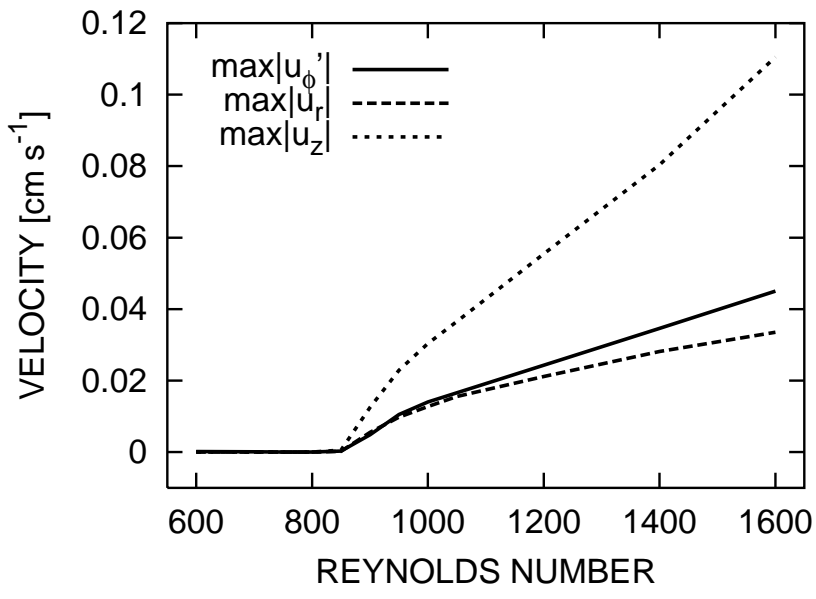

Fig. 4 Amplitudes of velocity as function of Reynolds number, $u_{\phi}^{\prime}$ is the deviation from the standard Couette solution. The amplitudes increase from the critical point $\operatorname{Re}_{c}=$ 842 and continue to increase with Re. Infinite cylinders, $\mathrm{Ha}=9.5, \hat{\mu}=0.27, \beta=4$.

\subsection{Frequencies}

Figure 2] shows snapshots of the streamfunction for the saturated state for Reynolds number just above the critical value, the three pairs of drifting vortices can be seen. This drift and its velocity agrees with the previous results, it is slow, for gallium the period of the traveling wave is about $230 \mathrm{~s}$ for $\beta=4$ which corresponds to $u_{\mathrm{drift}}=0.5 \mathrm{~mm} / \mathrm{s}$ (the period of one rotation of the inner cylinder is about $35 \mathrm{~s}$ for $\operatorname{Re}=900)$.

From the experimental point of view it is also convenient to present traveling wave frequencies as function of $\hat{\mu}$ (Fig. 3). All the frequencies were obtained by taking the spectrum of the velocity component $u_{z}(t)$ in the middle of the gap and then choosing the dominant frequency. Except for very small $\hat{\mu}$ the resulting frequencies are always smaller than the frequencies of both the cylinders. Beyond the Rayleigh limit (i.e. for $\hat{\mu}>0.25$ ) the characteristic frequency is about $10 \%$ of the frequency of the inner cylinder. That means that beyond the timescale of rotation another timescale exists in magnetic astrophysical systems which exceeds the rotation period by (say) a factor of ten. Due to the small electric conductivity, however, our TC-system only possesses a very short diffusion time of order of $10^{-2} \mathrm{~s}$.

\subsection{Velocity amplitudes and torque}

The saturated nonlinear solution provides the values of velocity amplitudes which have not been known before. The velocity component which is easiest to measure in the laboratory is $u_{z}$.

Figure 4 shows the dependence of different components of velocity as function of Reynolds number Re. The velocities are zero up to critical point $\mathrm{Re}_{\text {crit }}$ at which they start 


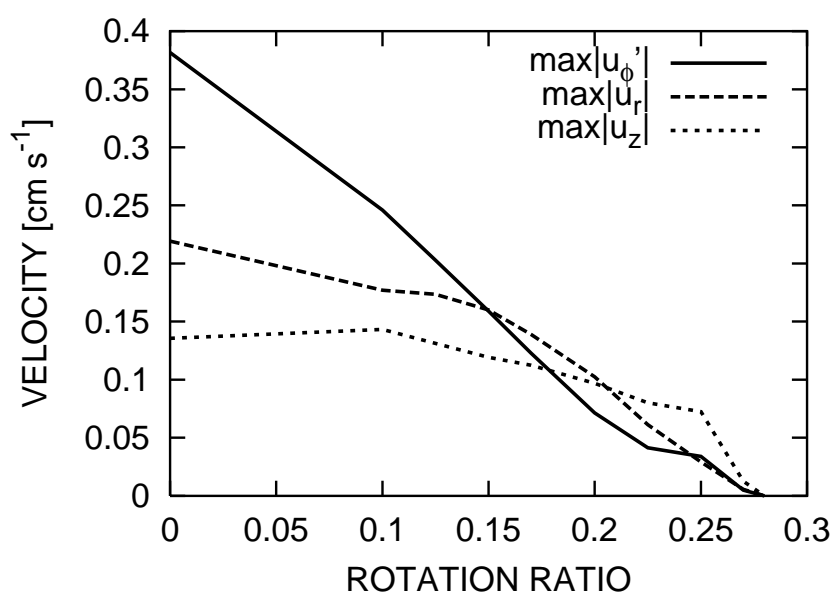

Fig. 5 Average maximum amplitudes of the velocity versus $\hat{\mu}$ for infinite cylinders, $\mathrm{Re}=900, \beta=4$, $\mathrm{Ha}=9.5$.

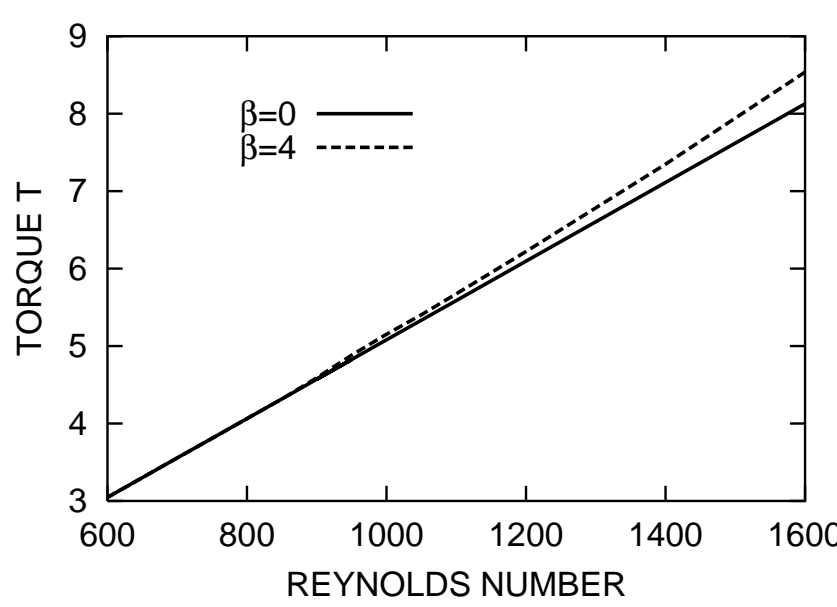

Fig. 6 Torque at the walls for two different $\beta$. Note that from the critical $\operatorname{Re}_{\text {crit }}=842$ for $\beta=4$ case the torque is larger than for the stable $\beta=0$ case, $\mathrm{Ha}=9.5$.

to grow. We notice that the values of $\boldsymbol{u}$ can be further increased by increasing the rotation rates of the container and therefore it is easier to measure $u_{z}$ for higher Re.

In Fig. 5 the maximum amplitudes of velocity for $\mathrm{Re}=$ 900 and $\beta=4$ are presented. For any stable solution all the amplitudes should be zero just beyond the Rayleigh line, i.e. on the right of $\hat{\mu}=0.25$. We see that with additional toroidal field this is not the case - the velocities for $\hat{\mu}=0.27$ are of order $0.1 \mathrm{~mm} / \mathrm{s}$ and drop here to zero for $\hat{\mu}=0.28$ for these parameters.

For MRI one expects the angular momentum to be transported outwards and this should result in an increase of the torque. We are mainly interested in torques at the walls at which the magnetic and advective torque vanish so the total torque is equal to the viscous one, which is defined as

$T(R)=-\int_{0}^{H} R^{3} \rho \nu \partial_{R}\left(\frac{u_{\phi}(R)}{R}\right) \mathrm{d} z$.
Although the change of the torque is rather small and would be difficult to measure in the laboratory, Fig. 6 shows that increase of the torque can be observed in our computations above the critical Reynolds number. Indeed the angular momentum is transported outwards.

\section{Finite container}

In any real experiment finite cylinders enclosed by endplates are used. It has been pointed out that the endplates can have significant impact on the flow especially for short cylinders and for fast rotation (e.g. Hollerbach \& Fournier 2004; Kageyama et al. 2004) also some solutions for reduction of the endplates effect have been proposed (Burin et al. 2006). We are mainly interested in knowing how the endplates and their mechanical and magnetic properties influence the velocity amplitudes and frequencies for slow rotating finite cylinders with aspect ratio of (say) $\Gamma=10$ and a current-free helical external field geometry.

\subsection{Ekman layer}

The existence of the viscous endplates results in developing so-called Ekman layers in which the velocity differs from any toroidal rotation as centrifugal force is weakened close to the vertical boundaries. This effect drives a global meridional circulation and two Ekman vortices appear. The velocity in this region depends on the Reynolds number and the velocity of the endplates themselves. As known for nonrotating endplates (or rotating as a solid body with $\Omega_{\text {out }}$ ) there is a radial inflow close to the boundaries and for solid-body rotation with $\Omega_{\text {in }}$ there is a radial outflow.

In the Rayleigh-stable regime the flow is hydrodynamically stable for both the infinite and finite containers, whereas for the latter two Ekman vortices are always present. We confirmed it with the numerical method that it is used here.

A simple estimate of the thickness of the Ekman layer (for endplates rigidly rotating with $\Omega_{\text {out }}$, see e.g. Ji et al. 2004) gives

$\delta \approx \sqrt{\frac{\nu}{\bar{\Omega}}} \approx 0.13 \mathrm{~cm}$

for the $\beta=4$ critical case where $\bar{\Omega}=\sqrt{\Omega_{\text {in }} \Omega_{\text {out }}}$. This is a larger value in comparison to other experiments dealing with faster rotation $\left(\mathrm{Re} \approx 10^{6}\right)$ in which case the possibility of developing hydrodynamical turbulence in the Ekman layer arises.

The typical velocity in the Ekman flow can be approximated by $\bar{u}_{\delta}=\sqrt{\mu \bar{\Omega}}$ which for $\operatorname{Re}=900$ with $0.02 \mathrm{~cm} / \mathrm{s}$ is of the same order for velocities for the unstable case presented for infinite cylinders (see Fig. 5). For Re $=900$ the maximum value of $u_{z}$ (in the corners) is above $0.1 \mathrm{~cm} \mathrm{~s}^{-1}$ which is ten times more than the values computed for infinite cylinders. Moreover, for the same parameters the Ekman circulation time $H / 2 \sqrt{\nu \bar{\Omega}}$ is of order $10^{3}$ s which is 


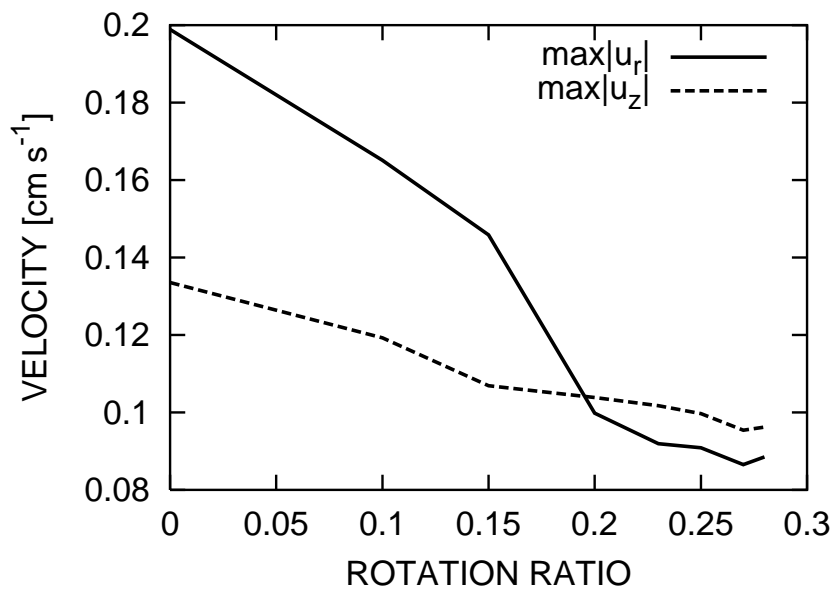

Fig. 7 The same as in Fig. 5 but for finite cylinders. $\operatorname{Re}=900, \beta=4, \mathrm{Ha}=9.5$, insulating endplates (pseudovacuum boundary conditions) both rotating with the outer cylinder.

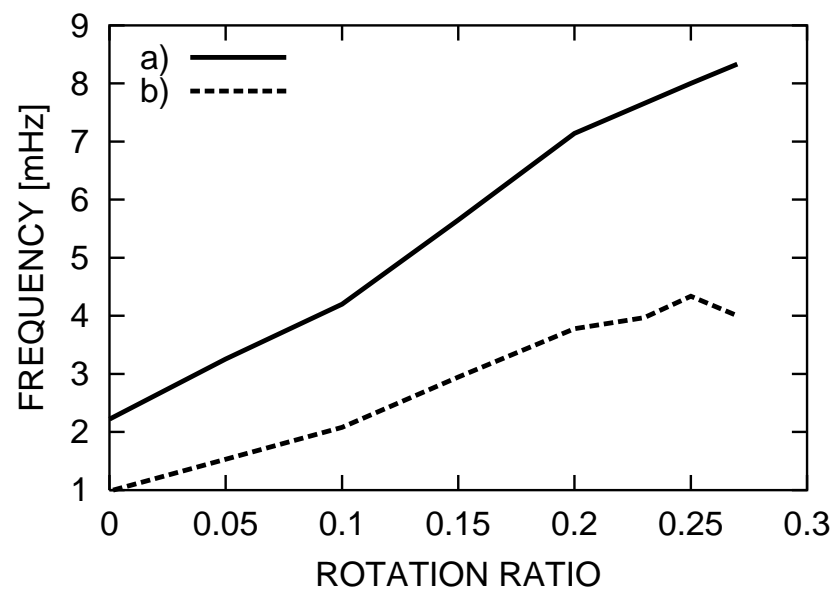

Fig. 8 Frequencies as function of rotation ratio $\hat{\mu}$ for finite cylinders. a) $\beta=6, \operatorname{Re}=1500, \mathrm{Ha}=9.52$, insulating endplates, bottom: fixed, upper: rotating with $\Omega_{\text {out }}$. b) $\beta=$ $4, \mathrm{Re}=900, \mathrm{Ha}=9.5$, insulating endplates rotating with $\Omega_{\text {out }}$.

comparable to typical MRI growth time. We must conclude that endplates can significantly alter the flow.

In the computations it turns out that in the presence of the external axial field, the Ekman flow induces significant $z$-gradients in $b_{\phi}$ which can result in a magnetic instability even for small Reynolds number. The critical numbers depend on magnetic properties of the endplates, their rotation and Hartmann number ${ }^{1}$. Since at this moment our aim is to investigate how the traveling-wave effects of the MRI with helical (current-free) field structure can be observed experimentally, this instability, although highly interesting, is beyond the scope of the present paper.

\footnotetext{
${ }^{1}$ e.g. $\mathrm{Re}_{\mathrm{crit}}=140$ for $\mathrm{Ha}=3.5$, perfectly conducting, fixed endplates
}

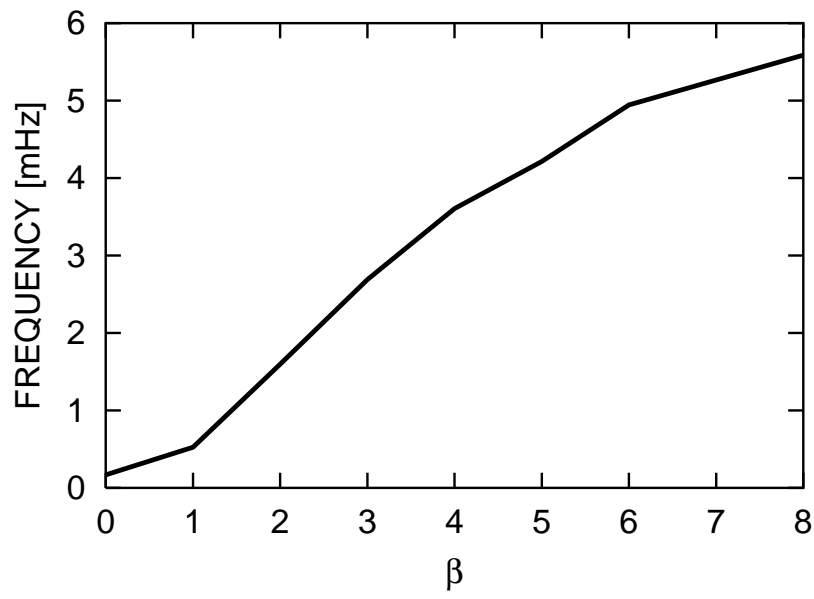

Fig.9 Frequencies of the traveling waves in the middle of the gap for finite cylinders as function of $\beta . \operatorname{Re}=900, \hat{\mu}=$ $0.27, \mathrm{Ha}=9.5$, insulating endplates rotating with $\Omega_{\text {out }}$.

\subsection{Amplitudes and frequencies}

The velocity amplitudes for finite cylinders just above the critical Reynolds number are shown in Fig. 7 We notice that due to the Ekman circulation those velocities do not drop to zero even for $\hat{\mu}=0.28$, which was true for infinite cylinders. Again the external toroidal field maintains traveling waves which can easily be observed in the laboratory. Analog to Fig. 3, Fig. 8 shows the frequencies of the velocity field in the center of the gap. Unlike for the infinite container, here the position of measurement does make difference since in the vicinity of the endplates the traveling wave is suppressed by the Ekman layer. From Fig. 9 we can clearly see that non-zero $\beta$ introduces vertical movement with very similar frequencies as for the infinite case (compare with $\hat{\mu}=0.27$ in Fig. 3).

Snapshots of streamfunction are shown in Fig. 10 We see that the flow is complicated and deformed when compared with infinite-container solutions. The $z$-asymmetry close to the endplates is due to different boundary conditions: the bottom endplate is at rest, the upper moves with the outer cylinder ${ }^{2}$. We conclude that even for nonsymmetric rotation of the endplates the traveling wave can still be observed.

Similarly like for the infinite case one can also observe the increase of torque in comparison with $\beta=0$ case (Fig. 11].

\section{Conclusions}

We have shown that, as predicted, additional toroidal fields reduce the critical Reynolds number for the MHD TaylorCouette flows for laboratory liquids with $\mathrm{Pm} \approx 10^{-5}$ $10^{-6}$. The nonlinear, axisymmetric simulations in the quasistatic approximation yield very similar characteristic values

2 This is the case for the current experimental setup of PROMISE 

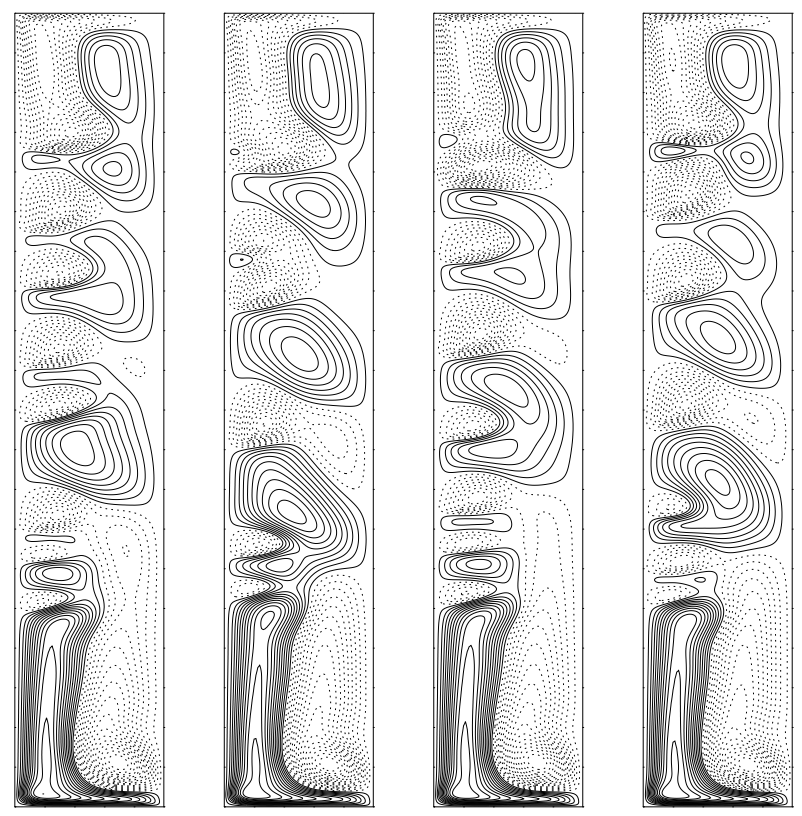

Fig. 10 Snapshots of contour lines of the streamfunction analog to Fig. 2 but for finite cylinders. $\operatorname{Re}=1500, \beta=$ $6, \mathrm{Ha}=9.5$, insulating endplates, bottom: fixed, upper: rotating with $\Omega_{\text {out }}$.

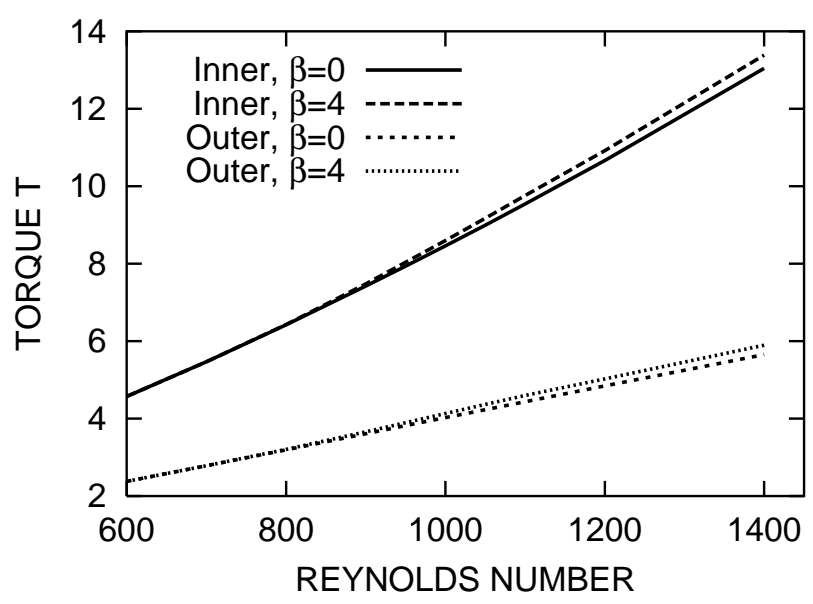

Fig. 11 Torque at the walls (inner and outer) for finite cylinders for different values of $\beta$. $\mathrm{Re}=900, \mathrm{Ha}=9.5$.

compared to those obtained with linear theory for infinite cylinders.

The velocity amplitudes which were not known until now, are small but still it is not very difficult to measure them experimentally. Similarly the velocity of the drift can easily be detected by looking at changes in the velocity field. We shall also mention that increasing Reynolds numbers result in linear (at least to some point) increase of velocity amplitudes, therefore it is easier to detect them for higher Re, however for too high rotation rates the assumption of axisymmetry may not be valid.
For finite aspect ratio calculations, the velocities induced by Ekman pumping are of similar order as those resulting from the instability. Therefore one should not neglect the influence of the endplates in such experiments. Nevertheless the influence of the toroidal field and the traveling wave can easily be detected in all cases.

Acknowledgements. J. Szklarski would like to thank A. Youd for providing the orginal version of the code and for the discussions.

\section{References}

Balbus, S. A., Hawley, J. F.: 1991, ApJ 376, 214

Burin, M., Ji, H., Schartman, E., Cutler, R., Heitzenroeder, P., Liu, W., Morris, L., Raftopolous, S.: 2006, Experiments in Fluids

Hollerbach, R., Fournier, A.: 2004, in: R. Rosner, G. Rüdiger, \& A. Bonanno (eds.), AIP Conf. Proc. 733: MHD Couette Flows: Experiments and Models, American Institute of Physics, New York, p. 114

Hollerbach, R., Rüdiger, G.: 2005, PhRvL 95, 124501

Ji, H., Goodman, J., Kageyama, A.: 2001, MNRAS 325, L1

Ji, H., Goodman, J., Kageyama, A., Burin, M., Schartman, E., Liu, W.: 2004, in: R. Rosner, G. Rüdiger, \& A. Bonanno (eds.), AIP Conf. Proc. 733: MHD Couette Flows: Experiments and Models, American Institute of Physics, New York, p. 21

Kageyama, A., Ji, H., Goodman, J., Chen, F., Shoshan, E.: 2004, arXiv:physics/0405123

Roberts, P. H.: 1967, An Introduction to Magnetohydrodynamics, Longmans, Greeen and Co. Ltd.

Rüdiger, G., Zhang, Y.: 2001, A\&A 378, 302

Rüdiger, G., Hollerbach, R., Schultz, M., Shalybkov, D. A.: 2005, AN 326, 409

Stefani, F., Gundrum, T., Gerbeth, G., Rüdiger, G., Schultz, M., Szklarski, J.: 2006, PhRvL (subm.)

Velikhov, E. P.: 1959, Sov. Phys. JETP 36, 995

Willis, A. P., Barenghi, C. F.: 2002, A\&A 388, 688

Youd, A. J., Barenghi, C. F.: 2006, JFM 550, 27

Zikanov, O., Thess, A.: 1998, JFM 358, 299 